\title{
Relationship between Individual Difference in Melatonin Suppression by Light and Habitual Bedtime
}

\author{
Shigekazu Higuchi ${ }^{1)}$, Yutaka Motohashi ${ }^{1)}$, Takafumi Maeda ${ }^{2)}$ and Keita Ishibashi ${ }^{3)}$ \\ 1) Department of Public Health, Akita University School of Medicine \\ 2) Department of Hygiene and Preventive Medicine, Fukushima Medical University School of Medicine \\ 3) Department of Human Living System Design, Faculty of Design, Kyushu University
}

\begin{abstract}
The purpose of this study was to determine the relationship between individual difference in melatonin suppression by exposure to light and habitual bedtime. Seventeen healthy male students (mean age: 22.6 $\pm 2.4 \mathrm{yr}$ ) volunteered to participate in the study. The subjects were exposed to light ( $1000 \mathrm{~lx})$ for 2 hours from 2 hours before the time of peak salivary melatonin concentration. Two hours after exposure to the light, melatonin suppression had occurred in fifteen subjects. No significant correlation was found between the rate of melatonin suppression and habitual bedtime in the fifteen subjects in whom melatonin suppression occurred. However, the habitual bedtime of the two subjects in whom melatonin suppression did not occur was earlier than that of the other subjects. These results suggest that there are some people with very low sensitivity to light and that this may affect habitual bedtime. J Physiol Anthropol Appl Human Sci 24(4): 419-423, 2005 http://www.jstage.jst.go.jp/browse/jpa [DOI: 10.2114/jpa.24.419]
\end{abstract}

Keywords: melatonin suppression, light, sensitivity, bedtime, sleep habits, physiological polymorphism

\section{Introduction}

Melatonin is secreted from the pineal gland during the night. This melatonin secretion is suppressed by exposure to a bright light (Lewy et al., 1980), and there is a dose-response relationship between light intensity and melatonin suppression (Aoki et al., 1998; Brainard et al., 1988; McIntyre et al., 1989; Zeitzer et al., 2000). There are individual differences in the magnitude of melatonin suppression in response to a given light intensity (Laakso et al., 1991, Smith et al., 2004). It has been reported that several factors such as recent history of exposure to light (Hébert et al., 2002; Smith et al., 2004), seasonal variation (Owen and Arendt, 1992), seasonal affective disorder (Nathan et al., 1999) and delayed sleep phase syndrome (DSPS) (Aoki et al., 2001) are related to individual differences in melatonin suppression. Aoki et al. (2001) reported that the magnitude of melatonin suppression by light in patients with delayed sleep phase syndrome (DSPS) was larger than that in healthy controls. The magnitude of melatonin suppression by light is expressed as "melatonin sensitivity to suppression by light". The results of that study suggest that hypersensitivity to light is related to delayed bedtime in patients with DSPS. However, there have been few studies in which the relationship between individual difference in melatonin sensitivity and habitual bedtime in normal healthy people has been examined.

More recent studies have shown that nocturnal melatonin secretion can be suppressed by exposure to a light of several hundred luxes, such as an ordinary room light (Aoki et al., 1998, Boivin et al., 1998; Higuchi et al., 2003; Zeitzer et al., 2000). It has been reported that endogenous melatonin secretion plays a role in gating nocturnal rise in sleep propensity (Lavie, 1997). These studies suggest that melatonin might be suppressed and nocturnal rise in sleep propensity reduced by even normal room light. Based on the results of these previous studies, it appears that individual difference in sensitivity of melatonin suppression to light affects habitual bedtime of even healthy people. In another words, people with a high sensitivity of melatonin suppression may have late habitual bedtime. Considering the increasing tendency of a night-shifted lifestyle in modern society, it would be worth examining the relationship between individual difference in magnitude of melatonin suppression and habitual bedtime.

The purpose of the present study was to investigate individual variation in melatonin suppression in healthy subjects and to examine the relationship between melatonin suppression by light and habitual bedtime in healthy subjects.

\section{Methods}

The study was conducted during the period from January to February. Twenty-one healthy male students (mean age: $22.4 \pm 2.4 \mathrm{yr}$ ) volunteered to participate in the study. Subjects 
gave written informed consent for participation in the study, which was approved by the Ethics Committee of Akita University School of Medicine. Before the experiment, each subject completed a questionnaire on morningnesseveningness preferences (ME score) (Horne and Östberg, 1976). Beginning 1 week prior to the start of the experiment, the subjects were instructed to keep their habitual sleep-wake rhythms and to conduct a self-recording of a sleep diary (bedtime, rising time, sleep quality, etc). The average bedtime and rising time on weekdays were used as habitual bedtime and rising time. The subjects were instructed not to spend much time outdoors in order to avoid exposure to bright light for a long time, since it has been reported that recent history of exposure to light affects the magnitude of melatonin suppression by light (Hébert et al., 2002; Smith et al., 2004). The daily activity pattern and light intensity to which each subject was exposed were recorded every minute using an accelerometer and optical sensor (Actiwatch-L, Mini-Mitter Co, Inc., USA) that was attached to the subject's wrist. There was no subject who was exposed to strong light intensity for a long time. They were also instructed not to consume caffeine and alcohol and not to perform intense exercise 24 hours prior to the experiments.

In the first experiment, each subject sat on a chair and spent 24 hours (9:00 to 9:00) in a dark experimental room $(<15 \mathrm{~lx})$, and saliva samples were collected every hour to determine peak salivary melatonin concentration. The subjects were allowed to watch a movie, listen to music and read a book. The saliva samples were kept frozen at $-30^{\circ} \mathrm{C}$ until analysis. After centrifugation (for 5 minutes at $3000 \mathrm{rpm}$ ), melatonin concentrations in the saliva samples were determined using a ELISA kit (Direct Saliva Melatonin ELISA, Bulmann, Switzerland) in our laboratory. After analyzing circadian variation in melatonin concentration, four subjects were excluded because their peak melatonin concentration could not be detected. Seventeen subjects (mean age: 22.6 $2.4 \mathrm{yr}$ ) completed the study.

The second experiment was conducted within 2 weeks after the first experiment in order to determine the magnitude of melatonin suppression by light. The protocol of the second experiment was based on that of a previous study (Aoki et al., 2001). The subjects were instructed to keep habitual sleepwake rhythm and to avoid exceptionally bright light in the morning and evening until the second experiment. The subjects came to the laboratory 5.5 hours before the time of peak melatonin concentration obtained in the first experiment and they were asked to sleep for 3 hours from 5 hours before the time of peak melatonin concentration. Then they were wakened and exposed to bright light (1000lx) for 2 hours using white fluorescent lamps (FRL-40S W/M 4500K, Matsushita Electric Industrial Co. Ltd., Osaka, Japan) placed on the ceiling. The light intensity was measured vertically at the subject's eye level using a light meter (CL-200, KONICA MINOLTA HOLDINGS, INC., Tokyo, Japan). During the exposure, each subject sat on a chair and watched a movie to fix his eyes. A 15 inch LCD display was placed about 2 meters in front of the subject. Saliva samples were collected before sleep and before exposure to the light and every hour during the period of exposure. The rate of suppression of melatonin concentration induced by the light was defined as [(melatonin concentration before exposure to light-melatonin concentration after exposure to light) / melatonin concentration before exposure to light] $\times 100$. Pearson's correlation coefficient was used to determine the relationships between variables.

\section{Results}

Data on habitual bedtime, rising time, and ME score are shown in Table 1. The average habitual bedtime and average rising time were 01:22 \pm 1.0 hours and 07:48 \pm 0.7 hours,

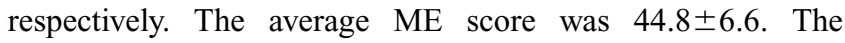
average time of the peak melatonin concentration was 04:14 \pm 1.6 hours.

Changes in salivary melatonin concentration after exposure to the light are shown in Table 1 and Fig. 1. Melatonin concentrations increased in all subjects during the sleep period. During the period of exposure to the light, melatonin suppression had occurred in fifteen subjects (solid lines), but not in two subjects (dotted lines) 2 hours after exposure to the light. For the subjects in whom melatonin suppression occurred $(n=15)$, the mean rates of melatonin suppression 1 hour and 2 hours after exposure were 43.6 \pm 20.8 and $62.9 \pm 20.5 \%$, respectively. The data for the two subjects without melatonin suppression were out of the distribution of data for the fifteen subjects with melatonin suppression, and these data were shown by using Grubbs Smirnov's method to be statistically outlying values (Fig. 1).

Figure 2 shows the relationship between rate of melatonin suppression 2 hours after the start of light exposure and habitual bedtime. No significant correlation was found between rate of melatonin suppression and habitual bedtime in fifteen subjects in whom melatonin suppression occurred $(r=0.07)$ (Fig. 2, solid circles), However, the bedtime of the two subjects in whom melatonin suppression did not occur (Fig. 2, open circles) was earlier than that of the other subjects (Fig. 2, solid circles). Rising time, ME score and time of peak melatonin concentration were not significantly correlated with rate of melatonin suppression.

\section{Discussion}

In a previous study using almost the same protocol as that used in the present study (light intensity, $10001 \mathrm{x}$; exposure duration, 2 hours; start time, 2 hours before peak melatonin), the average rate of melatonin suppression 2 hours after exposure was $55.5 \%$ and standard deviation was approximately $20 \%$ in healthy people (Aoki et al., 2001). These previously reported data are almost the same as the data obtained in the present study $(62.9 \pm 20.5 \%)$. Contrary to our expectation, there was no significant relationship between individual 
Table 1. Habitual bedtime, habitual rising time, ME score, time of peak melatonin concentration and rate of melatonin suppression

\begin{tabular}{cccccc}
\hline Subject & $\begin{array}{c}\text { Bed-time } \\
\text { (hour) }\end{array}$ & $\begin{array}{c}\text { Rising Time } \\
\text { (hour) }\end{array}$ & ME score & $\begin{array}{c}\text { Time of Peak } \\
\text { melatonin (hour) }\end{array}$ & $\begin{array}{c}\text { Melatonin } \\
\text { Suppression }(2 \mathrm{~h})(\%)\end{array}$ \\
\hline S 1 & $22: 38$ & $7: 13$ & 58 & $2: 00$ & -36.9 \\
S 2 & $23: 53$ & $6: 24$ & 50 & $3: 00$ & -21.5 \\
S 3 & $0: 40$ & $7: 05$ & 43 & $3: 00$ & 73.3 \\
S 4 & $0: 57$ & $8: 07$ & 42 & $6: 00$ & 67.9 \\
S 5 & $1: 05$ & $8: 54$ & 50 & $5: 00$ & 74.7 \\
S 6 & $1: 12$ & $8: 39$ & 37 & $4: 00$ & 76.9 \\
S 7 & $1: 27$ & $7: 47$ & 46 & $4: 00$ & 15.5 \\
S 8 & $1: 28$ & $8: 19$ & 44 & $5: 00$ & 84.1 \\
S 9 & $1: 37$ & $7: 25$ & 35 & $3: 00$ & 78.7 \\
S10 & $1: 34$ & $7: 40$ & 39 & $3: 00$ & 55.8 \\
S11 & $1: 34$ & $7: 47$ & 45 & $5: 00$ & 50.7 \\
S12 & $1: 40$ & $8: 31$ & 47 & $3: 00$ & 85.4 \\
S13 & $1: 46$ & $7: 57$ & 45 & $2: 00$ & 51.2 \\
S14 & $1: 45$ & $6: 55$ & 59 & $6: 00$ & 63.2 \\
S15 & $2: 30$ & $8: 18$ & 40 & $7: 00$ & 85.0 \\
S16 & $2: 38$ & $7: 40$ & 42 & $4: 00$ & $62.9^{*}$ \\
S17 & $2: 46$ & $7: 53$ & 40 & $1.6 \mathrm{~h}$ & $20.5^{*}$ \\
\hline Average & $1: 22$ & $7: 48$ & 44.8 & & \\
SD & $1.0 \mathrm{~h}$ & $0.7 \mathrm{~h}$ & 6.6 & & \\
\hline
\end{tabular}

* $\mathrm{n}=15$ (Data from two subjects in whom melatonin was not suppressed were excluded.)

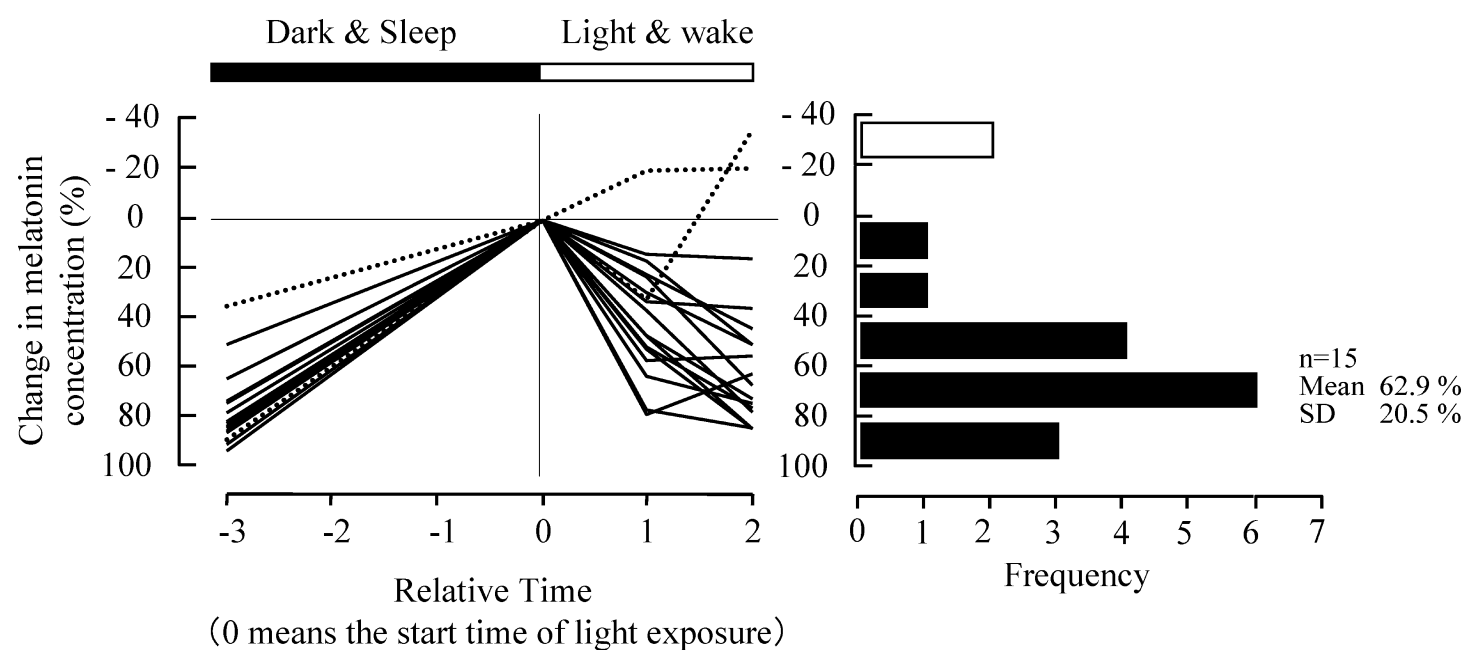

Fig. 1 Changes in melatonin concentration before and after exposure to light (Left) and frequency distribution of the rate of melatonin suppression 2 hours after exposure to light (Right). The rate of melatonin suppression was calculated based on the value before the start of exposure to the light (see text). The melatonin were suppressed (solid lines) in fifteen subjects, but the melatonin in two subjects were not suppressed 2 hours after exposure to the light (dotted lines). The data in two subjects with non-suppression of melatonin (Open bar) were out of the distribution of data for the fifteen subjects with suppression of melatonin (Solid bars).

difference in rate of melatonin suppression and habitual bedtime. This result suggests that individual difference in rate of melatonin suppression by a light does not affect habitual bedtime among people in whom melatonin secretion is suppressed by light. However, habitual bedtime might be affected more by melatonin suppression before going to bed. In the present study, as melatonin suppression was measured after habitual bed time of each subject, it is necessary to examine the relationship between habitual bedtime and melatonin suppression by light before habitual bedtime.

There were two subjects in whom melatonin suppression did not occur. This suggests that there are some people who have very low melatonin sensitivity to suppression by light. Furthermore, the two subjects in whom melatonin suppression did not occur had earlier habitual bedtimes than those of the subjects with suppression of melatonin. As for the causal relationship, it seems to be impossible that the habitual earlier bedtime causes non-suppression of melatonin, because it has 


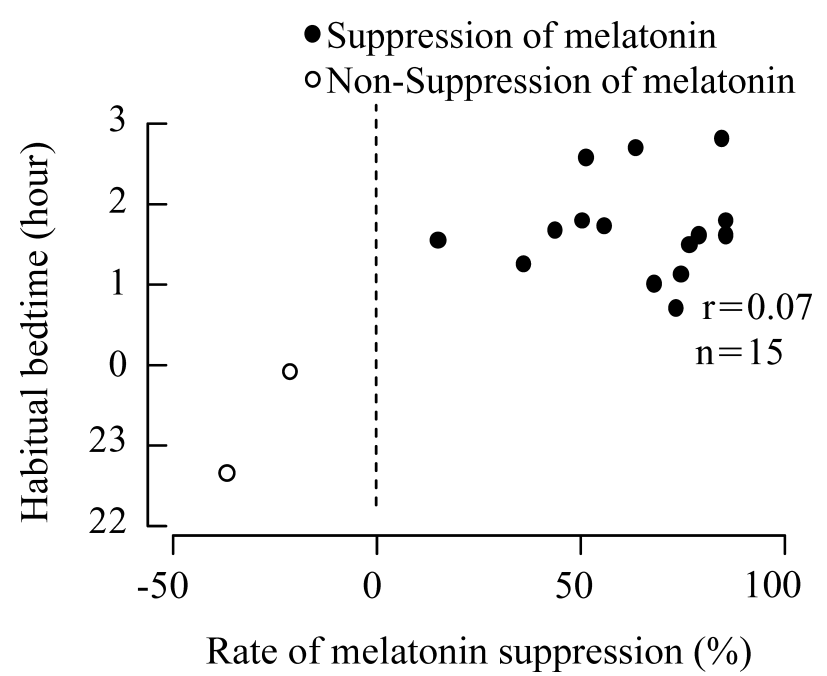

Fig. 2 Relationship between rate of melatonin suppression 2 hours after the start of light exposure and habitual bedtime. No significant correlation was found in fifteen subjects with suppression of melatonin ( $\mathrm{r}=0.07, \mathrm{n}=15)$ (solid circles). The two subjects (open circles) in whom melatonin was not suppressed had earlier habitual bedtimes.

been reported that melatonin suppression was observed in subjects with early habitual bedtime (before 00:00) (Aoki et al., 2001; Hébert et al., 2002; Owen and Arendt, 1992). One possible reason for the fact that there was no subject with melatonin suppression who had a habitual early bedtime (before 00:00) is selection bias in the present study. It was difficult to find subjects who have habitual early bedtime because almost all university students tend to go to bed late.

On the other hand, it may be possible that the physiological features of non-suppression of melatonin by light cause the habitual earlier bedtime. It has been reported that exogenous melatonin have hypnotic effects (Mishima et al., 1997; Nave et al., 1995). It is also thought that the endogenous melatonin opens the sleep gate at night (Dawson and Encel, 1993; Lavie, 1997; Wirz-Justice and Armstrong, 1996), while the hypnotic effects of endogenous melatonin have not been fully clarified (Murata et al., 1998). If endogenous melatonin does in fact have hypnotic effects, the physiological features of nonsuppression of melatonin by light could prevent the delay of bedtime. Further systematic study is needed to clarify the causal relationship between non-suppression of melatonin and early bedtime.

In the field of physiological anthropology, it is expected to investigate the variations in physiological responses from the viewpoint of polymorphism. The fact that there were two subjects in whom melatonin suppression did not occur by light may suggest the example of physiological polymorphism. It has been reported that melatonin secretion in three of twelve subjects was not suppressed by exposure to light (500 lx) for 3 hours (Hébert et al., 2002). A possible reason given for the non-suppression of melatonin is that the period of exposure to light was too early for the phase of melatonin secretion. In the present study, the effect of start time of light exposure on suppression of melatonin seems to be less because the start time of light exposure was individually set 2 hours before the time of peak melatonin concentration. However, it is not clear what mechanisms (genetic factors, environmental factors and cultural factors) are related to the low sensitivity of melatonin suppression to light. Further examination of more subjects in whom melatonin is not suppressed by light, determination of the causal relationships between non-suppression of melatonin and early bedtime and its mechanisms are needed.

Acknowledgement This study was supported by a Grantin-Aid for Scientific Research (S) (No. 15107006) from the Japanese Ministry of Education, Culture, Sports, Science, and Technology.

\section{References}

Aoki H, Ozeki Y, Yamada N (2001) Hypersensitivity of melatonin suppression in response to light in patients with delayed sleep phase syndrome. Chronobiol Int 18: 263-271

Aoki H, Yamada N, Ozeki Y, Yamane H, Kato N (1998) Minimum light intensity required to suppress nocturnal melatonin concentration in human saliva. Neurosci Lett 252: 91-94

Boivin DB, Czeisler CA (1998) Resetting of circadian melatonin and cortisol rhythms in humans by ordinary room light. Neuroreport 9: 779-782

Brainard GC, Lewy AJ, Menaker M, Fredrickson RH, Miller LS, Weleber RG, Cassone V, Hudson D (1988) Doseresponse relationship between light irradiance and the suppression of plasma melatonin in human volunteers. Brain Res 454: 212-218

Dawson D, Encel N (1993) Melatonin and sleep in humans. J Pineal Res 15: 1-12

Hébert M, Martin SK, Lee C, Eastman CI (2002) The effects of prior light history on the suppression of melatonin by light in humans. J Pineal Res 33: 198-203

Higuchi S, Motohashi Y, Liu Y, Ahara M, Kaneko Y (2003) Effects of VDT tasks with a bright display at night on melatonin, core temperature, heart rate, and sleepiness. J Appl Physiol 94: 1773-1776

Horne JA, Östberg O (1976) A self-assessment questionnaire to determine morningness-eveningness in human circadian rhythms. Int J Chronobiol 4: 97-110

Laakso ML, Porkka-Heiskanen T, Stenberg D, Alila A (1991) Interindividual differences in the responses of serum and salivary melatonin to light. In Fraschini F, Reiter RJ eds. Role of Melatonin and Pineal Peptides in Neuroimmunomodulation. Plenum Press, New York, 307-311

Lavie P (1997) Melatonin: role in gating nocturnal rise in sleep propensity. J Biol Rhythms 12: 657-665

Lewy AJ, Wehr TA, Goodwin FK, Newsome DA, Markey SP (1980) Light suppresses melatonin secretion in humans. 
Science 210: 1267-1269

McIntyre IM, Norman TR, Burrows GD, Armstrong SM (1989) Human melatonin suppression by light is intensity dependent. J Pineal Res 6: 149-156

Mishima K, Satoh K, Shimizu T, Hishikawa Y (1997) Hypnotic and hypothermic action of daytime-administered melatonin. Psychopharmacol (Berl) 133: 168-171

Murata J, Sawamura Y, Ikeda J, Hashimoto S, Honma K (1998) Twenty-four hour rhythm of melatonin in patients with a history of pineal and/or hypothalamo-neurohypophyseal germinoma. J Pineal Res 25: 159-166

Nathan PJ, Burrows GD, Norman TR (1999) Melatonin sensitivity to dim white light in affective disorders. Neuropsychopharmacol 21: 408-413

Nave R, Peled R, Lavie P (1995) Melatonin improves evening napping. Eur J Pharmacol 275: 213-216

Owen J, Arendt J (1992) Melatonin suppression in human subjects by bright and dim light in antarctica: time and season-dependent effects. Neurosci Lett 137: 181-184
Smith KA, Schoen MW, Czeisler CA (2004) Adaptation of human pineal melatonin suppression by recent photic history. J Clin Endocrinol Metab 89: 3610-3614

Wirz-Justice A, Armstrong SM (1996) Melatonin: nature's soporific? J Sleep Res 5: 137-141

Zeitzer JM, Dijk DJ, Kronauer R, Brown E, Czeisler C (2000) Sensitivity of the human circadian pacemaker to nocturnal light: melatonin phase resetting and suppression. J Physiol 526 (Pt 3): 695-702

Received: January 31, 2005

Accepted: March 9, 2005

Correspondence to: Shigekazu Higuchi, Ph.D., Department of Public Health, Akita University School of Medicine, 1-1-1 Hondo, Akita 010-8543, Japan

Phone: +81-18-884-6087

Fax: +81-18-836-2609

e-mail: higuchi@med.akita-u.ac.jp 